\title{
Exact solution of the $s p(4)$ integrable spin chain with generic boundaries
}

\author{
Guang-Liang Li, ${ }^{a}$ Junpeng Cao,,${ }^{b, c, d}$ Panpan Xue, ${ }^{a}$ Zhi-Rong Xin, ${ }^{e}$ Kun Hao, ${ }^{e, f}$ \\ Wen-Li Yang, ${ }^{e, f, g, 1}$ Kangjie Shi ${ }^{e}$ and Yupeng Wang ${ }^{b, d, 1}$ \\ ${ }^{a}$ Department of Applied Physics, Xian Jiaotong University, \\ Xian 710049, China \\ ${ }^{b}$ Beijing National Laboratory for Condensed Matter Physics, Institute of Physics, \\ Chinese Academy of Sciences, \\ Beijing 100190, China \\ ${ }^{c}$ Songshan Lake Materials Laboratory, \\ Dongguan, Guangdong 523808, China \\ ${ }^{d}$ School of Physical Sciences, University of Chinese Academy of Sciences, \\ Beijing, China \\ ${ }^{e}$ Institute of Modern Physics, Northwest University, \\ Xian 710127, China \\ ${ }^{f}$ Shaanxi Key Laboratory for Theoretical Physics Frontiers, \\ Xian 710127, China \\ ${ }^{g}$ School of Physics, Northwest University, \\ Xian 710127, China \\ E-mail: leegl@xjtu.edu.cn, junpengcao@iphy.ac.cn, panpanxue66@163.com, \\ zhirongxin@foxmail.com, haoke72@163.com, wlyang@nwu.edu.cn, \\ kjshi@nwu.edu.cn, yupeng@iphy.ac.cn
}

ABSTRACT: The off-diagonal Bethe ansatz method is generalized to the integrable model associated with the $s p(4)$ (or $C_{2}$ ) Lie algebra. By using the fusion technique, we obtain the complete operator product identities among the fused transfer matrices. These relations, together with some asymptotic behaviors and values of the transfer matrices at certain points, enable us to determine the eigenvalues of the transfer matrices completely. For the periodic boundary condition case, we recover the same $T-Q$ relations obtained via conventional Bethe ansatz methods previously, while for the off-diagonal boundary condition case, the eigenvalues are given in terms of inhomogeneous $T-Q$ relations, which could not be obtained by the conventional Bethe ansatz methods. The method developed in this paper can be directly generalized to generic $s p(2 n)$ (i.e., $C_{n}$ ) integrable model.

Keywords: Bethe Ansatz, Lattice Integrable Models

ARXIV EPRINT: 1812.03618

\footnotetext{
${ }^{1}$ Corresponding author.
} 


\section{Contents}

1 Introduction 1

2 Periodic $s p(4)$-invariant spin chain $\quad 2$

2.1 The system 2

2.2 Fusion 3

$2.3 T-Q$ relations 5

3 Off-diagonal integrable open boundary case $\quad 8$

3.1 Open chain 8

3.2 Fusion of the reflection matrices $\quad 9$

$\begin{array}{ll}3.3 & \text { Operator product identities } \\ \end{array}$

$\begin{array}{lll}3.4 & \text { Inhomogeneous } T-Q \text { relations } & 12\end{array}$

4 Discussion $\quad 15$

\section{Introduction}

Quantum integrable models play important roles in a variety of fields such as quantum field theory, condensed matter physics and statistical physics, because they can provide solid benchmarks for understanding the many-body effects and new physical concepts in corresponding universal classes [1-5].

Solving integrable models without U(1) symmetry had been an interesting issue and attracted a lot of attentions in the past several decades [6-17]. Recently, a generic method (the off-diagonal Bethe ansatz (ODBA)) for solving the integrable models with or without obvious reference states was proposed [18]. With the help of the proposed inhomogeneous $T-Q$ relations, several typical models without $\mathrm{U}(1)$ symmetry were solved exactly [19]. Based on the eigenvalues obtained via ODBA, the corresponding Bethe-type eigenstates were also retrieved $[20,21]$. The nested ODBA was first proposed to study the quantum spin chain model related to $A_{n}$ algebra [22]. However, its generalization to integrable models associated with other high-rank Lie algebras such as $B_{n}, C_{n}$ and $D_{n}$ are still missing. In this paper, we generalize the nested ODBA method to $C_{2}$ spin chain model with both periodic and off-diagonal open boundary conditions. This method can also be applied to generic $s p(2 n)$ quantum integrable spin chains.

The paper is organized as follows. In section 2, we study the $C_{2}$ model with periodic boundary condition. The closed functional relations to determine the eigenvalues of the transfer matrix are obtained by the fusion technique. The exact solutions we derived coincide exactly with those obtained by the conventional Bethe Ansatz methods [23, 24]. The exact spectrum of the $C_{2}$ model with off-diagonal open boundary conditions, which 
could not be solved via either algebraic or analytic Bethe Ansatz, is given in terms of an inhomogeneous $T-Q$ relation in section 3. Section 4 is attributed to the concluding remarks.

\section{Periodic $s p(4)$-invariant spin chain}

\subsection{The system}

Let $\mathrm{V}$ denote a 4 -dimensional linear space with an orthonormal basis $\{|i\rangle \mid i=1, \cdots, 4\}$ which endows the fundamental representation of the $C_{2}$ algebra. The $s p(4)$-invariant $R$ matrix $R(u) \in \operatorname{End}(\mathrm{V} \otimes \mathrm{V})$ is given by its matrix elements [24]

$$
R_{12}(u)_{k l}^{i j}=u(u+3) \delta_{i k} \delta_{j l}+(u+3) \delta_{i l} \delta_{j k}-u \xi_{i} \xi_{k} \delta_{j \bar{i}} \delta_{k \bar{l}},
$$

where the index $\bar{i}$ is defined by $i+\bar{i}=5, \xi_{i}=1$ if $i \in\{1,2\}$ and $\xi_{i}=-1$ if $i \in\{3,4\}$. Let us take the notations for simplicity

$$
\begin{aligned}
R_{12}(u)_{i i}^{i i} & =a(u)=(u+1)(u+3), \\
R_{12}(u)_{i j}^{i j} & =b(u)=u(u+3), \quad i \neq j, \bar{j}, \\
R_{12}(u)_{\bar{i} i}^{i \bar{i}}=c(u) & =2 u+3, \\
\xi_{i} \xi_{j} R_{12}(u)_{j \bar{j}}^{i \bar{i}} & =d(u)=-u, \quad i \neq j, \bar{j}, \\
R_{12}(u)_{i \bar{i}}^{i \bar{i}}=e(u) & =u(u+2), \\
R_{12}(u)_{j i}^{i j} & =g(u)=u+3, \quad i \neq j, \bar{j} .
\end{aligned}
$$

The $R$-matrix (2.1) enjoys the properties:

$$
\begin{aligned}
\text { regularity : } & R_{12}(0)=\rho_{1}(0)^{\frac{1}{2}} \mathcal{P}_{12}, \\
\text { unitarity : } & R_{12}(u) R_{21}(-u)=\rho_{1}(u), \\
\text { crossing - unitarity : } & R_{12}(u)^{t_{1}} R_{21}(-u-6)^{t_{1}}=\rho_{1}(u+3),
\end{aligned}
$$

where $\rho_{1}(u)=a(u) a(-u), \mathcal{P}$ is the permutation operator with the elements $\mathcal{P}_{k l}^{i j}=\delta_{i l} \delta_{j k}$, and $t_{i}$ denotes the transposition in $i$-th space, $R_{21}(u)=\mathcal{P}_{12} R_{12}(u) \mathcal{P}_{12}$. Here and below we adopt the standard notation: for any matrix $A \in \operatorname{End}(\mathrm{V}), A_{j}$ is an embedding operator in the tensor space $\mathrm{V} \otimes \mathrm{V} \otimes \cdots$, which acts as $A$ on the $j$-th space and as an identity on the other factor spaces; $R_{i j}(u)$ is an embedding operator of $R$-matrix in the tensor space, which acts as an identity on the factor spaces except for the $i$-th and $j$-th ones. The $R$-matrix satisfies the quantum Yang-Baxter equation (QYBE)

$$
R_{12}(u-v) R_{13}(u) R_{23}(v)=R_{23}(v) R_{13}(u) R_{12}(u-v) .
$$

Let us introduce the "row-to-row" (or one-row ) monodromy matrix $T(u)$, which is a $4 \times 4$ matrix with operator-valued elements acting on $\mathrm{V}^{\otimes N}$,

$$
T_{0}(u)=R_{01}\left(u-\theta_{1}\right) R_{02}\left(u-\theta_{2}\right) \cdots R_{0 N}\left(u-\theta_{N}\right),
$$


where $\left\{\theta_{j} \mid j=1, \cdots, N\right\}$ are arbitrary free complex parameters usually called as inhomogeneous parameters. The transfer matrix $t_{p}(u)$ of the associated spin chain with the periodic boundary condition is given by [5]

$$
t_{p}(u)=\operatorname{tr}_{0} T_{0}(u)
$$

The QYBE (2.3) of the $R$-matrix implies that one-row monodromy matrix $T(u)$ satisfies the Yang-Baxter relation

$$
R_{12}(u-v) T_{1}(u) T_{2}(v)=T_{2}(v) T_{1}(u) R_{12}(u-v) .
$$

From the above relation, one can prove that the transfer matrices with different spectral parameters commute with each other, $\left[t_{p}(u), t_{p}(v)\right]=0$. Then $t_{p}(u)$ serves as the generating functional of the conserved quantities, which ensures the integrability of the $s p(4)$-invariant spin chain with the periodic boundary condition described by the Hamiltonian

$$
H_{p}=\left.\frac{\partial \ln t_{p}(u)}{\partial u}\right|_{u=0,\left\{\theta_{j}\right\}=0}=\sum_{k=1}^{N} H_{k k+1},
$$

where $H_{k k+1}=\left.\mathcal{P}_{k k+1} R_{k k+1}^{\prime}(u)\right|_{u=0}$. The periodic boundary condition implies $H_{N N+1}$ $=H_{N 1}$.

\subsection{Fusion}

The $R$-matrix (2.1) may degenerate to projection operators at some special points of $u$, which makes it possible for us to do fusion [25-31]. For example, if $u=-3$, we have

$$
R_{12}(-3)=P_{12}^{(1)} \times S_{1} .
$$

Here $P_{12}^{(1)}$ is a one-dimensional projection operator with the form

$$
P_{12}^{(1)}=\left|\psi_{0}\right\rangle\left\langle\psi_{0}\right|
$$

where $\left|\psi_{0}\right\rangle=\frac{1}{2}(|14\rangle+|23\rangle-|32\rangle-|41\rangle)$ is a vector in the space $\mathrm{V} \otimes \mathrm{V}$ and $S_{1}$ is a constant matrix (we omit its expression because we do not need it). If $u=-1$, then

$$
R_{12}(-1)=P_{12}^{(5)} \times S_{2} .
$$

Here $P_{12}^{(5)}$ is a five-dimensional projection operator with the form

$$
P_{12}^{(5)}=\sum_{i=1}^{5}\left|\psi_{i}^{(5)}\right\rangle\left\langle\psi_{i}^{(5)}\right|,
$$

where the corresponding vectors are

$$
\begin{array}{rlrl}
\left|\psi_{1}^{(5)}\right\rangle & =\frac{1}{\sqrt{2}}(|12\rangle-|21\rangle), & \left|\psi_{2}^{(5)}\right\rangle=\frac{1}{\sqrt{2}}(|13\rangle-|31\rangle), \\
\left|\psi_{3}^{(5)}\right\rangle=\frac{1}{2}(|14\rangle-|41\rangle+|32\rangle-|23\rangle), & \left|\psi_{4}^{(5)}\right\rangle=\frac{1}{\sqrt{2}}(|24\rangle-|42\rangle), \\
\left|\psi_{5}^{(5)}\right\rangle=\frac{1}{\sqrt{2}}(|34\rangle-|43\rangle), &
\end{array}
$$

and $S_{2}$ is a constant matrix. 
From the QYBE (2.3), the one-dimensional fusion associated with the projector (2.9) leads to

$$
\begin{aligned}
& P_{12}^{(1)} R_{23}(u) R_{13}(u-3) P_{12}^{(1)}=a(u) e(u-3) P_{12}^{(1)}, \\
& P_{21}^{(1)} R_{32}(u) R_{31}(u-3) P_{21}^{(1)}=a(u) e(u-3) P_{21}^{(1)} .
\end{aligned}
$$

From the five-dimensional fusion associated with the projector (2.11), we obtain a new fused $\bar{R}$-matrix

$$
\begin{aligned}
& \bar{R}_{\langle 12\rangle 3}(u)=\left[\left(u+\frac{3}{2}\right) \tilde{\rho}_{0}\left(u+\frac{1}{2}\right)\right]^{-1} P_{12}^{(5)} R_{23}\left(u+\frac{1}{2}\right) R_{13}\left(u-\frac{1}{2}\right) P_{12}^{(5)} \\
& \bar{R}_{3\langle 12\rangle}(u)=\left[\left(u+\frac{3}{2}\right) \tilde{\rho}_{0}\left(u+\frac{1}{2}\right)\right]^{-1} P_{21}^{(5)} R_{32}\left(u+\frac{1}{2}\right) R_{31}\left(u-\frac{1}{2}\right) P_{21}^{(5)}
\end{aligned}
$$

where $\tilde{\rho}_{0}(u)=(u-1)(u+3)$. For simplicity, let us denote the resulting five-dimensional fusion space by $\overline{\mathrm{V}}_{\overline{1}}$ which is spanned by $\left\{\left|\psi_{i}^{(5)}\right\rangle \mid i=1, \ldots, 5\right\}$. It is easy to check that the matrix elements of the fused $R$-matrix $\bar{R}_{\overline{1} 3}(u) \equiv \bar{R}_{\langle 12\rangle}(u)$ (or $\bar{R}_{3 \overline{1}}(u) \equiv \bar{R}_{3,\langle 12\rangle}(u)$ ), as functions of $u$, are degree-one polynomials of $u$. Moreover, we have

$$
\begin{aligned}
\bar{R}_{\overline{1} 2}(u) \bar{R}_{2 \overline{1}}(-u) & =-\left(u+\frac{5}{2}\right)\left(u-\frac{5}{2}\right), \\
\bar{R}_{\overline{1} 2}(u)^{t_{1}} \bar{R}_{2 \overline{1}}(-u-6)^{t_{1}} & =-\left(u+\frac{1}{2}\right)\left(u+\frac{11}{2}\right), \\
\bar{R}_{\overline{1} 2}(u-v) \bar{R}_{\overline{1} 3}(u) R_{23}(v) & =R_{23}(v) \bar{R}_{\overline{1} 3}(u) \bar{R}_{\overline{1} 2}(u-v) .
\end{aligned}
$$

At the point of $u=-\frac{5}{2}$, the fused $\bar{R}$-matrix reduces to a four-dimensional projector

$$
\bar{R}_{\overline{1} 2}\left(-\frac{5}{2}\right)=P_{\overline{1} 2}^{(4)} \times \bar{S}
$$

where $\bar{S}$ is a constant matrix and the four-dimensional projector $P_{\overline{1} 2}^{(4)}$ takes the form of

$$
P_{\overline{1} 2}^{(4)}=\sum_{i=1}^{4}\left|\psi_{i}^{(4)}\right\rangle\left\langle\psi_{i}^{(4)}\right|,
$$

with the corresponding vectors as

$$
\begin{aligned}
\left|\psi_{1}^{(4)}\right\rangle & =\frac{1}{\sqrt{5}}\left(\sqrt{2}\left|\psi_{1}^{(5)}\right\rangle \otimes|3\rangle-\sqrt{2}\left|\psi_{2}^{(5)}\right\rangle \otimes|2\rangle-\left|\psi_{3}^{(5)}\right\rangle \otimes|1\rangle\right), \\
\left|\psi_{2}^{(4)}\right\rangle & =\frac{1}{\sqrt{5}}\left(-\sqrt{2}\left|\psi_{1}^{(5)}\right\rangle \otimes|4\rangle-\sqrt{2}\left|\psi_{4}^{(5)}\right\rangle \otimes|1\rangle+\left|\psi_{3}^{(5)}\right\rangle \otimes|2\rangle\right), \\
\left|\psi_{3}^{(4)}\right\rangle & =\frac{1}{\sqrt{5}}\left(-\sqrt{2}\left|\psi_{2}^{(5)}\right\rangle \otimes|4\rangle-\sqrt{2}\left|\psi_{5}^{(5)}\right\rangle \otimes|1\rangle+\left|\psi_{3}^{(5)}\right\rangle \otimes|3\rangle\right), \\
\left|\psi_{4}^{(4)}\right\rangle & =\frac{1}{\sqrt{5}}\left(-\sqrt{2}\left|\psi_{4}^{(5)}\right\rangle \otimes|3\rangle+\sqrt{2}\left|\psi_{5}^{(5)}\right\rangle \otimes|2\rangle-\left|\psi_{3}^{(5)}\right\rangle \otimes|4\rangle\right) .
\end{aligned}
$$


The property (2.15) allow us to do fusion with $P_{\overline{1} 2}^{(4)}$ again. The results read

$$
\begin{aligned}
& R_{\langle\overline{1} 2\rangle 3}(u)=(u+5)^{-1} P_{\overline{1} 2}^{(4)} R_{23}(u+2) \bar{R}_{\overline{1} 3}\left(u-\frac{1}{2}\right) P_{\overline{1} 2}^{(4)}, \\
& R_{3\langle\overline{1} 2\rangle}(u)=(u+5)^{-1} P_{2 \overline{1}}^{(4)} R_{32}(u+2) \bar{R}_{3 \overline{1}}\left(u-\frac{1}{2}\right) P_{2 \overline{1}}^{(4)} .
\end{aligned}
$$

After taking the correspondence

$$
\left|\psi_{i}^{(4)}\right\rangle \longrightarrow|i\rangle, \quad i=1, \ldots, 4,
$$

we get the key relations

$$
R_{\langle\overline{1} 2\rangle{ }_{3}}(u)=R_{13}(u), \quad R_{3\langle\overline{1} 2\rangle}(u)=R_{31}(u),
$$

where the $R$-matrices $R_{13}(u)$ and $R_{31}(u)$ are given by (2.1).

\section{$2.3 \quad T-Q$ relations}

From the fused $\bar{R}$-matrix, we can define the fused monodromy matrix

$$
\bar{T}_{\overline{0}}(u)=\bar{R}_{\overline{0} 1}\left(u-\theta_{1}\right) \bar{R}_{\overline{0} 2}\left(u-\theta_{2}\right) \cdots \bar{R}_{\overline{0} N}\left(u-\theta_{N}\right),
$$

which is a $5 \times 5$ matrix with operator-valued elements acting on $\mathrm{V}^{\otimes N}$. The fused $\bar{R}$-matrix and the fused monodromy matrix $\bar{T}(u)$ satisfy the Yang-Baxter relation

$$
\bar{R}_{\overline{1} 2}(u-v) \bar{T}_{\overline{1}}(u) T_{2}(v)=T_{2}(v) \bar{T}_{\overline{1}}(u) \bar{R}_{\overline{1} 2}(u-v) .
$$

The fused transfer matrix is given by

$$
\bar{t}_{p}(u)=\operatorname{tr}_{\overline{0}} \bar{T}_{\overline{0}}(u)
$$

Using fusion relations (2.12), (2.13) and (2.17), we have

$$
\begin{aligned}
P_{21}^{(1)} T_{1}(u) T_{2}(u-3) P_{21}^{(1)} & =\prod_{i=1}^{N} a\left(u-\theta_{i}\right) e\left(u-\theta_{i}-3\right) P_{21}^{(1)}, \\
P_{21}^{(5)} T_{1}(u) T_{2}(u-1) P_{21}^{(5)} & =\prod_{i=1}^{N}\left(u-\theta_{i}+1\right) \tilde{\rho}_{0}\left(u-\theta_{i}\right) \bar{T}_{\langle 12\rangle}\left(u-\frac{1}{2}\right), \\
P_{\overline{1} 2}^{(4)} T_{2}(u) \bar{T}_{\overline{1}}\left(u-\frac{5}{2}\right) P_{\overline{1} 2}^{(4)} & =\prod_{i=1}^{N}\left(u-\theta_{i}+3\right) T_{\langle\overline{1} 2\rangle}(u-2) .
\end{aligned}
$$

Following the method developed in [22] and using the identity (2.19), we can show that the following identities hold

$$
\begin{aligned}
T_{1}\left(\theta_{j}\right) T_{2}\left(\theta_{j}-3\right) & =P_{21}^{(1)} T_{1}\left(\theta_{j}\right) T_{2}\left(\theta_{j}-3\right), \\
T_{1}\left(\theta_{j}\right) T_{2}\left(\theta_{j}-1\right) & =P_{21}^{(5)} T_{1}\left(\theta_{j}\right) T_{2}\left(\theta_{j}-1\right), \\
T_{2}\left(\theta_{j}\right) \bar{T}_{\overline{1}}\left(\theta_{j}-\frac{5}{2}\right) & =P_{\overline{1} 2}^{(4)} T_{2}\left(\theta_{j}\right) \bar{T}_{\overline{1}}\left(\theta_{j}-\frac{5}{2}\right) .
\end{aligned}
$$


Considering the relations (2.19) and (2.23)-(2.28), we obtain the operator identities among the fused transfer matrices as

$$
\begin{aligned}
t_{p}\left(\theta_{j}\right) t_{p}\left(\theta_{j}-3\right) & =\prod_{i=1}^{N} a\left(\theta_{j}-\theta_{i}\right) e\left(\theta_{j}-\theta_{i}-3\right), \\
t_{p}\left(\theta_{j}\right) t_{p}\left(\theta_{j}-1\right) & =\prod_{i=1}^{N}\left(\theta_{j}-\theta_{i}+1\right) \tilde{\rho}_{0}\left(\theta_{j}-\theta_{i}\right) \bar{t}_{p}\left(\theta_{j}-\frac{1}{2}\right), \\
t_{p}\left(\theta_{j}\right) \bar{t}_{p}\left(\theta_{j}-\frac{5}{2}\right) & =\prod_{i=1}^{N}\left(\theta_{j}-\theta_{i}+3\right) t_{p}\left(\theta_{j}-2\right) .
\end{aligned}
$$

The commutativity of the transfer matrices $t_{p}(u)$ and $\bar{t}_{p}(u)$ with different spectral parameters implies that they have common eigenstates (namely, the common eigenstates do not depend on the spectral parameter $u$ ). Let us denote the eigenvalues of the transfer matrices $t_{p}(u)$ and $\bar{t}_{p}(u)$ as $\Lambda_{p}(u)$ and $\bar{\Lambda}_{p}(u)$, respectively. From the identities (2.29)-(2.31), we have

$$
\begin{aligned}
\Lambda_{p}\left(\theta_{j}\right) \Lambda_{p}\left(\theta_{j}-3\right) & =\prod_{i=1}^{N} a\left(\theta_{j}-\theta_{i}\right) e\left(\theta_{j}-\theta_{i}-3\right), \\
\Lambda_{p}\left(\theta_{j}\right) \Lambda_{p}\left(\theta_{j}-1\right) & =\prod_{i=1}^{N}\left(\theta_{j}-\theta_{i}+1\right) \tilde{\rho}_{0}\left(\theta_{j}-\theta_{i}\right) \bar{\Lambda}_{p}\left(\theta_{j}-\frac{1}{2}\right), \\
\Lambda_{p}\left(\theta_{j}\right) \bar{\Lambda}_{p}\left(\theta_{j}-\frac{5}{2}\right) & =\prod_{i=1}^{N}\left(\theta_{j}-\theta_{i}+3\right) \Lambda_{p}\left(\theta_{j}-2\right) .
\end{aligned}
$$

The eigenvalue $\Lambda_{p}(u)$ of the transfer matrix $t_{p}(u)$ is a degree $2 N$ polynomial of $u$, which can be completely determined by $2 N+1$ conditions. Besides the functional relations (2.32)(2.34), we still need one more condition which can be obtained by analyzing the asymptotic behavior of $t_{p}(u)$. From the definition, the asymptotic behavior of $t_{p}(u)$ can be calculated as

$$
\left.t_{p}(u)\right|_{u \rightarrow \infty}=4 u^{2 N} \times \operatorname{id}+\cdots,
$$

which leads to

$$
\left.\Lambda_{p}(u)\right|_{u \rightarrow \infty}=4 u^{2 N}+\cdots
$$

The eigenvalue $\bar{\Lambda}_{p}(u)$ of the fused transfer matrix $\bar{t}_{p}(u)$ is a degree $N$ polynomial of $u$, which can be completely determined by the functional relations (2.32)-(2.34) and the asymptotic behavior of $\bar{t}_{p}(u)$ given by

$$
\left.\bar{t}_{p}(u)\right|_{u \rightarrow \infty}=5 u^{N} \times \mathrm{id}+\cdots,
$$

or

$$
\left.\bar{\Lambda}_{p}(u)\right|_{u \rightarrow \infty}=5 u^{N}+\cdots .
$$


Thus the $3 N+2$ relations $(2.32)-(2.36)$ completely determine the eigenvalues $\Lambda_{p}(u)$ and $\bar{\Lambda}_{p}(u)$, which are given in terms of the homogeneous $T-Q$ relations:

$$
\begin{aligned}
\Lambda_{p}(u)= & Z_{1}^{(p)}(u)+Z_{2}^{(p)}(u)+Z_{3}^{(p)}(u)+Z_{4}^{(p)}(u), \\
\bar{\Lambda}_{p}(u)= & \prod_{i=1}^{N}\left[\left(u-\theta_{i}+\frac{3}{2}\right) \tilde{\rho}_{0}\left(u-\theta_{i}+\frac{1}{2}\right)\right]^{-1}\left\{Z _ { 1 } ^ { ( p ) } ( u + \frac { 1 } { 2 } ) \left[Z_{2}^{(p)}\left(u-\frac{1}{2}\right)+Z_{3}^{(p)}\left(u-\frac{1}{2}\right)\right.\right. \\
& \left.\left.+Z_{4}^{(p)}\left(u-\frac{1}{2}\right)\right]+\left[Z_{2}^{(p)}\left(u+\frac{1}{2}\right)+Z_{3}^{(p)}\left(u+\frac{1}{2}\right)\right] Z_{4}^{(p)}\left(u-\frac{1}{2}\right)\right\},
\end{aligned}
$$

where

$$
\begin{aligned}
Z_{1}^{(p)}(u) & =\prod_{j=1}^{N} a\left(u-\theta_{j}\right) \frac{Q_{p}^{(1)}(u-1)}{Q_{p}^{(1)}(u)}, \\
Z_{2}^{(p)}(u) & =\prod_{j=1}^{N} b\left(u-\theta_{j}\right) \frac{Q_{p}^{(1)}(u+1) Q_{p}^{(2)}\left(u-\frac{3}{2}\right)}{Q_{p}^{(1)}(u) Q_{p}^{(2)}\left(u+\frac{1}{2}\right)} \\
Z_{3}^{(p)}(u) & =\prod_{j=1}^{N} b\left(u-\theta_{j}\right) \frac{Q_{p}^{(1)}(u+1) Q_{p}^{(2)}\left(u+\frac{5}{2}\right)}{Q_{p}^{(1)}(u+2) Q_{p}^{(2)}\left(u+\frac{1}{2}\right)}, \\
Z_{4}^{(p)}(u) & =\prod_{j=1}^{N} e\left(u-\theta_{j}\right) \frac{Q_{p}^{(1)}(u+3)}{Q_{p}^{(1)}(u+2)} \\
Q_{p}^{(m)}(u) & =\prod_{k=1}^{L_{m}}\left(u-\mu_{k}^{(m)}+\frac{m}{2}\right), \quad m=1,2 .
\end{aligned}
$$

Because the eigenvalues $\Lambda_{p}(u)$ and $\bar{\Lambda}_{p}(u)$ must be polynomials of $u$, the residues of right hand sides of equations (2.37)-(2.38) should be zero, which gives rise to the constraints of the Bethe roots $\left\{\mu_{k}^{(m)}\right\}$, namely, these parameters should satisfy the Bethe Ansatz equations

$$
\begin{array}{ll}
\frac{Q_{p}^{(1)}\left(\mu_{k}^{(1)}+\frac{1}{2}\right) Q_{p}^{(2)}\left(\mu_{k}^{(1)}-2\right)}{Q_{p}^{(1)}\left(\mu_{k}^{(1)}-\frac{3}{2}\right) Q_{p}^{(2)}\left(\mu_{k}^{(1)}\right)}=-\prod_{j=1}^{N} \frac{\mu_{k}^{(1)}+\frac{1}{2}-\theta_{j}}{\mu_{k}^{(1)}-\frac{1}{2}-\theta_{j}}, & k=1, \cdots, L_{1}, \\
\frac{Q_{p}^{(2)}\left(\mu_{l}^{(2)}+1\right) Q_{p}^{(1)}\left(\mu_{l}^{(2)}-\frac{3}{2}\right)}{Q_{p}^{(2)}\left(\mu_{l}^{(2)}-3\right) Q_{p}^{(1)}\left(\mu_{l}^{(2)}+\frac{1}{2}\right)}=-1, & l=1, \cdots, L_{2} .
\end{array}
$$

We note that the Bethe ansatz equations obtained from the regularity of $\Lambda_{p}(u)$ are the same as those obtained from the regularity of $\bar{\Lambda}_{p}(u)$. It is easy to check that $\Lambda_{p}(u)$ and $\bar{\Lambda}_{p}(u)$ satisfy the functional relations $(2.32)-(2.34)$ and the asymptotic behaviors (2.35) and (2.36). Therefore, we conclude that $\Lambda_{p}(u)$ and $\bar{\Lambda}_{p}(u)$ are the eigenvalues of the transfer matrices $t_{p}(u)$ and $\bar{t}_{p}(u)$, respectively. It is remarked that the $T-Q$ relation (2.37) and the associated Bethe ansatz equations (2.40)-(2.41)(after taking the homogeneous limit $\left.\left\{\theta_{j} \rightarrow 0 \mid j=1,2, \cdots, N\right\}\right)$ coincide with those obtained previously via conventional Bethe ansatz methods [23, 24] . 
The eigenvalues of the Hamiltonian (2.7) then can be expressed in terms of the Bethe roots as

$$
E_{p}=\left.\frac{\partial \ln \Lambda_{p}(u)}{\partial u}\right|_{u=0,\left\{\theta_{j}\right\}=0}
$$

\section{Off-diagonal integrable open boundary case}

\subsection{Open chain}

Integrable open chain can be constructed as follows $[3,4]$. Let us introduce a pair of $K$-matrices $K^{-}(u)$ and $K^{+}(u)$. The former satisfies the reflection equation (RE)

$$
R_{12}(u-v) K_{1}^{-}(u) R_{21}(u+v) K_{2}^{-}(v)=K_{2}^{-}(v) R_{12}(u+v) K_{1}^{-}(u) R_{21}(u-v),
$$

and the latter satisfies the dual RE

$$
\begin{aligned}
& R_{12}(-u+v) K_{1}^{+}(u) R_{21}(-u-v-6) K_{2}^{+}(v) \\
& \quad=K_{2}^{+}(v) R_{12}(-u-v-6) K_{1}^{+}(u) R_{21}(-u+v) .
\end{aligned}
$$

For open spin-chains, instead of the standard "row-to-row" monodromy matrix $T(u)(2.4)$, one needs to consider the "double-row" monodromy matrix as follows. Besides the monodromy matrix $T_{0}(u)$ given by (2.4), we also need a crossed monodromy matrix

$$
\hat{T}_{0}(u)=R_{N 0}\left(u+\theta_{N}\right) \cdots R_{20}\left(u+\theta_{2}\right) R_{10}\left(u+\theta_{1}\right),
$$

which satisfies the Yang-Baxter relation

$$
R_{12}(u-v) \hat{T}_{1}(u) \hat{T}_{2}(v)=\hat{T}_{2}(v) \hat{T}_{1}(u) R_{12}(u-v)
$$

The transfer matrix $t(u)$ is defined as [4]

$$
t(u)=\operatorname{tr}_{0}\left\{K_{0}^{+}(u) T_{0}(u) K_{0}^{-}(u) \hat{T}_{0}(u)\right\} .
$$

From the Yang-Baxter relation, reflection equation and dual reflection equation, one can prove that the transfer matrices with different spectral parameters commute with each other, $[t(u), t(v)]=0$. Therefore, $t(u)$ serves as the generating function of all the conserved quantities of the system. The associated quantum spin chain with integrable boundary interactions is given by the Hamiltonian

$$
\begin{aligned}
H & =\left.\frac{1}{2} \frac{\partial \ln t(u)}{\partial u}\right|_{u=0,\left\{\theta_{j}\right\}=0} \\
& =\sum_{k=1}^{N-1} H_{k k+1}+\frac{K_{1}^{-\prime}(0)}{2 \zeta}+\frac{t r_{0}\left\{K_{0}^{+}(0) H_{N 0}\right\}}{t r_{0} K_{0}^{+}(0)} .
\end{aligned}
$$

In this paper, we consider the open chain with off-diagonal $K$-matrix $K^{-}(u)$ [32-34]

$$
K^{-}(u)=\zeta+M u, \quad M=\left(\begin{array}{cccc}
-1 & 0 & c_{1} & 0 \\
0 & -1 & 0 & c_{1} \\
c_{2} & 0 & 1 & 0 \\
0 & c_{2} & 0 & 1
\end{array}\right)
$$


while the dual reflection matrix $K^{+}(u)$ is

$$
K^{+}(u)=\left.K^{-}(-u-3)\right|_{\zeta, c_{i} \rightarrow \tilde{\zeta}, \tilde{c}_{i}}
$$

Here $\zeta, c_{1}, c_{2}$ and $\tilde{\zeta}, \tilde{c}_{1}, \tilde{c}_{2}$ are some generic parameters describing the boundary fields applied on the end sites. It is easily to check that $\left[K^{-}(u), K^{+}(v)\right] \neq 0$, which implies that the $K^{ \pm}(u)$ matrices cannot be diagonalized simultaneously. In this case, it is quite hard to derive solutions via conventional Bethe Ansatz methods due to the absence of a proper reference state. We will generalize the method developed in section 2 to get eigenvalues of the transfer matrix $t(u)(3.5)$ specified by the $K$-matrices (3.7) and (3.8) in the following subsections.

\subsection{Fusion of the reflection matrices}

In order to obtain closed operator identities, we study first fusions of the reflection matrices. The one-dimensional fusion for the reflection matrices gives

$$
\begin{aligned}
P_{21}^{(1)} K_{1}^{-}(u) R_{21}(2 u-3) K_{2}^{-}(u-3) P_{12}^{(1)} & =(u-1)(u-3) h(u) P_{12}^{(1)}, \\
P_{12}^{(1)} K_{2}^{+}(u-3) R_{12}(-2 u-3) K_{1}^{+}(u) P_{21}^{(1)} & =(u+1)(u+3) \tilde{h}(u) P_{21}^{(1)},
\end{aligned}
$$

where

$$
\begin{aligned}
& h(u)=4\left[\left(1+c_{1} c_{2}\right) u^{2}-\zeta^{2}\right], \\
& \tilde{h}(u)=4\left[\left(1+\tilde{c}_{1} \tilde{c}_{2}\right) u^{2}-\tilde{\zeta}^{2}\right] .
\end{aligned}
$$

From the five-dimensional fusion, we obtain a new fused reflection matrices $\bar{K}$ as

$$
\begin{aligned}
& \bar{K}_{\langle 12\rangle}^{-}(u)=[(2 u-1)(2 u+3)]^{-1} P_{21}^{(5)} K_{1}^{-}\left(u+\frac{1}{2}\right) R_{21}(2 u) K_{2}^{-}\left(u-\frac{1}{2}\right) P_{12}^{(5)}, \\
& \bar{K}_{\langle 12\rangle}^{+}(u)=[(2 u+3)(2 u+7)]^{-1} P_{12}^{(5)} K_{2}^{+}\left(u-\frac{1}{2}\right) R_{12}(-2 u-6) K_{1}^{+}\left(u+\frac{1}{2}\right) P_{21}^{(5)},
\end{aligned}
$$

where the projector $P_{12}^{(5)}$ is given by (2.11). Due to the dimension of the projected space $\overline{\mathrm{V}}$ is 5 , the corresponding $\bar{K}_{\overline{1}}^{ \pm}(u)$ are both $5 \times 5$ matrices and their elements are all degree two polynomials of $u$. The fused $\bar{R}$-matrix and the fused reflection matrix $\bar{K}^{ \pm}(u)$ satisfy the reflection equations

$$
\begin{aligned}
& \bar{R}_{\overline{1} 2}(u-v) \bar{K}_{\overline{1}}^{-}(u) \bar{R}_{2 \overline{1}}(u+v) K_{2}^{-}(v)=K_{2}^{-}(v) \bar{R}_{\overline{1} 2}(u+v) \bar{K}_{\overline{1}}^{-}(u) \bar{R}_{2 \overline{1}}(u-v), \\
& \begin{array}{r}
\bar{R}_{\overline{1} 2}(-u+v) \bar{K}_{\overline{1}}^{+}(u) \bar{R}_{2 \overline{1}}(-u-v-6) K_{2}^{+}(v) \\
=K_{2}^{+}(v) \bar{R}_{\overline{1} 2}(-u-v-6) \bar{K}_{\overline{1}}^{+}(u) \bar{R}_{2 \overline{1}}(-u+v) .
\end{array}
\end{aligned}
$$

Now let us turn to the fusion between the reflection matrices $K^{ \pm}(u)$ and $\bar{K}^{ \pm}(u)$ by the four-dimensional projector $P_{\overline{1} 2}^{(4)}$ given by $(2.16)$, which gives

$$
\begin{aligned}
& K_{\langle\overline{1} 2\rangle}^{-}(u)=4[(2 u-1) h(u+2)]^{-1} P_{\overline{1} 2}^{(4)} K_{2}^{-}(u+2) \bar{R}_{\overline{1} 2}\left(2 u+\frac{3}{2}\right) \bar{K}_{\overline{1}}^{-}\left(u-\frac{1}{2}\right) P_{2 \overline{1}}^{(4)}, \\
& K_{\langle\overline{1} 2\rangle}^{+}(u)=-2[(u+5) \tilde{h}(u+2)]^{-1} P_{2 \overline{1}}^{(4)} \bar{K}_{\overline{1}}^{+}\left(u-\frac{1}{2}\right) \bar{R}_{2 \overline{1}}\left(-2 u-\frac{15}{2}\right) K_{2}^{+}(u+2) P_{\overline{1} 2}^{(4)} .
\end{aligned}
$$


Both $K_{\langle\overline{1} 2\rangle}^{ \pm}(u)$ are $4 \times 4$ matrices, whose matrix elements are degree one polynomials of $u$. Moreover, keeping the correspondence (2.18) in mind, we have

$$
K_{\langle\overline{1} 2\rangle}^{ \pm}(u) \equiv K^{ \pm}(u)
$$

where the $K$-matrices $K^{ \pm}(u)$ are given by (3.7) and (3.8).

\subsection{Operator product identities}

For the open boundary case, besides the fused monodromy matrix $\bar{T}_{\overline{0}}(u)$ given by $(2.20)$, we also need the reflecting fused monodromy matrix $\hat{\bar{T}}(u)$ given by

$$
\hat{\bar{T}}_{\overline{0}}(u)=\bar{R}_{N \overline{0}}\left(u+\theta_{N}\right) \cdots \bar{R}_{2 \overline{0}}\left(u+\theta_{2}\right) \bar{R}_{1 \overline{0}}\left(u+\theta_{1}\right),
$$

where the dimension of auxiliary space $\overline{\mathrm{V}}$ is 5 and the quantum space keeps unchanged. The matrix $\hat{\bar{T}}_{\overline{0}}$ satisfies the Yang-Baxter relation

$$
\bar{R}_{\overline{1} 2}(u-v) \hat{\bar{T}}_{\overline{1}}(u) \hat{T}_{2}(v)=\hat{T}_{2}(v) \hat{\bar{T}}_{\overline{1}}(u) \bar{R}_{\overline{1} 2}(u-v) .
$$

The fused transfer matrix $\bar{t}(u)$ is

$$
\bar{t}(u)=\operatorname{tr}_{\overline{0}}\left\{\bar{K}_{\overline{0}}^{+}(u) \bar{T}_{\overline{0}}(u) \bar{K}_{\overline{0}}^{-}(u) \hat{\bar{T}}_{\overline{0}}(u)\right\} .
$$

From the definitions (3.5) and (3.18), the transfer matrix $t(u)$ (resp. $\bar{t}(u)$ ), as a function of $u$, is a polynomial of degree $4 N+2$ (resp. a polynomial with degree of $2 N+4$ ). In order to determine the eigenvalues of the transfer matrices $t(u)$ and $\bar{t}(u)$, one needs at least $6 N+8$ conditions.

With the fusion relations (2.12), (2.13) and (2.17), we have

$$
\begin{aligned}
P_{12}^{(1)} \hat{T}_{1}(u) \hat{T}_{2}(u-3) P_{12}^{(1)} & =\prod_{i=1}^{N} a\left(u+\theta_{i}\right) e\left(u+\theta_{i}-3\right) P_{12}^{(1)}, \\
P_{12}^{(5)} \hat{T}_{1}(u) \hat{T}_{2}(u-1) P_{12}^{(5)} & =\prod_{i=1}^{N}\left(u+\theta_{i}+1\right) \tilde{\rho}_{0}\left(u+\theta_{i}\right) \hat{\bar{T}}_{\langle 12\rangle}\left(u-\frac{1}{2}\right), \\
P_{2 \overline{1}}^{(4)} \hat{T}_{2}(u) \hat{\bar{T}}_{\overline{1}}\left(u-\frac{5}{2}\right) P_{2 \overline{1}}^{(4)} & =\prod_{i=1}^{N}\left(u+\theta_{i}+3\right) \hat{T}_{\langle\overline{1} 2\rangle}(u-2) .
\end{aligned}
$$

We can show that Yang-Baxter relations (3.4) and (3.17) at certain points also give

$$
\begin{aligned}
\hat{T}_{1}\left(-\theta_{j}\right) \hat{T}_{2}\left(-\theta_{j}-\frac{3}{2}\right) & =P_{12}^{(1)} \hat{T}_{1}\left(-\theta_{j}\right) \hat{T}_{2}\left(-\theta_{j}-\frac{3}{2}\right), \\
\hat{T}_{1}\left(-\theta_{j}\right) \hat{T}_{2}\left(-\theta_{j}-1\right) & =P_{12}^{(5)} \hat{T}_{1}\left(-\theta_{j}\right) \hat{T}_{2}\left(-\theta_{j}-1\right), \\
\hat{T}_{2}\left(-\theta_{j}\right) \hat{\bar{T}}_{\overline{1}}\left(-\theta_{j}-\frac{5}{2}\right) & =P_{2 \overline{1}}^{(4)} \hat{T}_{2}\left(-\theta_{j}\right) \hat{\bar{T}}_{\overline{1}}\left(-\theta_{j}-\frac{5}{2}\right) .
\end{aligned}
$$


Keeping the identity (2.19) in mind and using the relations (2.23)-(2.28), and (3.19)-(3.24), we obtain

$$
\begin{aligned}
& t\left( \pm \theta_{j}\right) t\left( \pm \theta_{j}-3\right)=\frac{1}{2^{4}} \frac{\left( \pm \theta_{j}-1\right)\left( \pm \theta_{j}-3\right)\left( \pm \theta_{j}+1\right)\left( \pm \theta_{j}+3\right)}{\left( \pm \theta_{j}-\frac{3}{2}\right)\left( \pm \theta_{j}-\frac{1}{2}\right)\left( \pm \theta_{j}+\frac{3}{2}\right)\left( \pm \theta_{j}+\frac{1}{2}\right)} \\
& \times h\left( \pm \theta_{j}\right) \tilde{h}\left( \pm \theta_{j}\right) \prod_{i=1}^{N} a\left( \pm \theta_{j}-\theta_{i}\right) a\left( \pm \theta_{j}+\theta_{i}\right) e\left( \pm \theta_{j}-\theta_{i}-3\right) e\left( \pm \theta_{j}+\theta_{i}-3\right) \\
& t\left( \pm \theta_{j}\right) t\left( \pm \theta_{j}-1\right)= \frac{\left( \pm \theta_{j}-1\right)\left( \pm \theta_{j}+1\right)\left( \pm \theta_{j}+1\right)\left( \pm \theta_{j}+3\right)}{\left( \pm \theta_{j}-\frac{1}{2}\right)\left( \pm \theta_{j}+\frac{1}{2}\right)\left( \pm \theta_{j}+\frac{3}{2}\right)\left( \pm \theta_{j}+\frac{5}{2}\right)} \\
& \times \prod_{i=1}^{N}\left( \pm \theta_{j}-\theta_{i}+1\right)\left( \pm \theta_{j}+\theta_{i}+1\right) \tilde{\rho}_{0}\left( \pm \theta_{j}-\theta_{i}\right) \tilde{\rho}_{0}\left( \pm \theta_{j}+\theta_{i}\right) \bar{t}\left( \pm \theta_{j}-\frac{1}{2}\right) \\
& t\left( \pm \theta_{j}\right) \bar{t}\left( \pm \theta_{j}-\frac{5}{2}\right)= \frac{1}{2^{4}} \frac{\left( \pm \theta_{j}-\frac{5}{2}\right)\left( \pm \theta_{j}+3\right)}{\left( \pm \theta_{j}-1\right)\left( \pm \theta_{j}+\frac{3}{2}\right)} h\left( \pm \theta_{j}\right) \tilde{h}\left( \pm \theta_{j}\right) \\
& \times \prod_{i=1}^{N}\left( \pm \theta_{j}-\theta_{i}+3\right)\left( \pm \theta_{j}+\theta_{i}+3\right) t\left( \pm \theta_{j}-2\right)
\end{aligned}
$$

From the definition, the asymptotic behavior of $t(u)$ can be calculated as

$$
\left.t(u)\right|_{u \rightarrow \infty}=-\operatorname{tr} \tilde{M} M \times u^{4 N+2} \times \mathrm{id}+\cdots,
$$

where $\tilde{M}=\left.M\right|_{\zeta, c_{1}, c_{2} \rightarrow \tilde{\zeta}, \tilde{c}_{1}, \tilde{c}_{2}}$. Direct calculation gives

$$
\operatorname{tr} \tilde{M} M=4+2 c_{1} \tilde{c}_{2}+2 c_{2} \tilde{c}_{1}
$$

Besides, we also know the values of $t(u)$ at the points of 0 and -3 ,

$$
\begin{gathered}
t(0)=\operatorname{tr}\left\{K^{+}(0)\right\} \zeta \prod_{i=1}^{N} \rho_{1}\left(-\theta_{i}\right) \times \mathrm{id}, \\
t(-3)=\operatorname{tr}\left\{K^{-}(-3)\right\} \tilde{\zeta} \prod_{i=1}^{N} \rho_{1}\left(-\theta_{i}\right) \times \mathrm{id} .
\end{gathered}
$$

The asymptotic behavior of $\bar{t}(u)$ reads

$$
\left.\bar{t}(u)\right|_{u \rightarrow \infty}=\operatorname{tr}_{12} P_{12}^{(5)}(\tilde{M} M)_{1}(\tilde{M} M)_{2} P_{12}^{(5)} \times u^{2 N+4} \times \mathrm{id}+\cdots
$$

Direct calculation shows

$$
\operatorname{tr}_{12} P_{12}^{(5)}(\tilde{M} M)_{1}(\tilde{M} M)_{2} P_{12}^{(5)}=\left(2+c_{1} \tilde{c}_{2}+c_{2} \tilde{c}_{1}\right)^{2}+\left(1+c_{1} c_{2}\right)\left(1+\tilde{c}_{1} \tilde{c}_{2}\right) .
$$


Using the method developed in [22], we can evaluate the values of $\bar{t}(u)$ at some special points as follows:

$$
\begin{aligned}
\bar{t}(0) & =\frac{5}{2^{4}}\left(1+c_{1} c_{2}-4 \zeta^{2}\right)\left(1+\tilde{c}_{1} \tilde{c}_{2}-4 \tilde{\zeta}^{2}\right) \prod_{i=1}^{N}\left(\frac{5}{2}-\theta_{i}\right)\left(\frac{5}{2}+\theta_{i}\right) \times \mathrm{id}, \\
\bar{t}(-3) & =\frac{5}{2^{4}}\left(1+c_{1} c_{2}-4 \zeta^{2}\right)\left(1+\tilde{c}_{1} \tilde{c}_{2}-4 \tilde{\zeta}^{2}\right) \prod_{i=1}^{N}\left(\frac{5}{2}-\theta_{i}\right)\left(\frac{5}{2}+\theta_{i}\right) \times \mathrm{id}, \\
\bar{t}\left(-\frac{1}{2}\right) & =\frac{5}{4} \frac{\zeta \tilde{\zeta}}{\prod_{i=1}^{N}\left(1-\theta_{i}\right)\left(1+\theta_{i}\right)} t(-1), \\
\bar{t}\left(-\frac{5}{2}\right) & =\frac{5}{4} \frac{\zeta \tilde{\zeta}}{\prod_{i=1}^{N}\left(1-\theta_{i}\right)\left(1+\theta_{i}\right)} t(-2) .
\end{aligned}
$$

\subsection{Inhomogeneous $T-Q$ relations}

So far we have obtained the $6 N+8$ conditions (3.25)-(3.36), which allow us to determine the eigenvalues of the transfer matrices $t(u)$ and $\bar{t}(u)$. It is easy to show that the transfer matrix $t(u)$ and its fused one $\bar{t}(u)$ satisfy the commutation relations

$$
[t(u), t(v)]=[\bar{t}(u), \bar{t}(v)]=[t(u), \bar{t}(v)]=0 .
$$

Let $|\Psi\rangle$ be a common eigenstate of the transfer matrices with the eigenvalues $\Lambda(u)$ and $\bar{\Lambda}(u)$

$$
t(u)|\Psi\rangle=\Lambda(u)|\Psi\rangle, \quad \bar{t}(u)|\Psi\rangle=\bar{\Lambda}(u)|\Psi\rangle .
$$

From the identities (3.25)-(3.27), we obtain the following closed functional relations

$$
\begin{aligned}
\Lambda\left( \pm \theta_{j}\right) \Lambda\left( \pm \theta_{j}-3\right) & =\frac{1}{2^{4}} \frac{\left( \pm \theta_{j}-1\right)\left( \pm \theta_{j}-3\right)\left( \pm \theta_{j}+1\right)\left( \pm \theta_{j}+3\right)}{\left( \pm \theta_{j}-\frac{3}{2}\right)\left( \pm \theta_{j}-\frac{1}{2}\right)\left( \pm \theta_{j}+\frac{3}{2}\right)\left( \pm \theta_{j}+\frac{1}{2}\right)} \\
\times h\left( \pm \theta_{j}\right) \tilde{h}\left( \pm \theta_{j}\right) & \prod_{i=1}^{N} a\left( \pm \theta_{j}-\theta_{i}\right) a\left( \pm \theta_{j}+\theta_{i}\right) e\left( \pm \theta_{j}-\theta_{i}-3\right) e\left( \pm \theta_{j}+\theta_{i}-3\right) \\
\Lambda\left( \pm \theta_{j}\right) \Lambda\left( \pm \theta_{j}-1\right) & =\frac{\left( \pm \theta_{j}-1\right)\left( \pm \theta_{j}+1\right)\left( \pm \theta_{j}+1\right)\left( \pm \theta_{j}+3\right)}{\left( \pm \theta_{j}-\frac{1}{2}\right)\left( \pm \theta_{j}+\frac{1}{2}\right)\left( \pm \theta_{j}+\frac{3}{2}\right)\left( \pm \theta_{j}+\frac{5}{2}\right)} \\
\times \prod_{i=1}^{N}\left( \pm \theta_{j}-\theta_{i}+1\right)\left( \pm \theta_{j}+\theta_{i}+1\right) \tilde{\rho}_{0}\left( \pm \theta_{j}-\theta_{i}\right) \tilde{\rho}_{0}\left( \pm \theta_{j}+\theta_{i}\right) \bar{\Lambda}\left( \pm \theta_{j}-\frac{1}{2}\right) & \\
\Lambda\left( \pm \theta_{j}\right) \bar{\Lambda}\left( \pm \theta_{j}-\frac{5}{2}\right) & =\frac{1}{2^{4}} \frac{\left( \pm \theta_{j}-\frac{5}{2}\right)\left( \pm \theta_{j}+3\right)}{\left( \pm \theta_{j}-1\right)\left( \pm \theta_{j}+\frac{3}{2}\right)} h\left( \pm \theta_{j}\right) \tilde{h}\left( \pm \theta_{j}\right) \\
& \times \prod_{i=1}^{N}\left( \pm \theta_{j}-\theta_{i}+3\right)\left( \pm \theta_{j}+\theta_{i}+3\right) \Lambda\left( \pm \theta_{j}-2\right) \\
\left.\Lambda(u)\right|_{u \rightarrow \infty} & =-\left(4+2 c_{1} \tilde{c}_{2}+2 c_{2} \tilde{c}_{1}\right) u^{4 N+2}+\cdots \\
\Lambda(0) & =4 \zeta \tilde{\zeta} \prod_{i=1}^{N} \rho_{1}\left(-\theta_{i}\right)
\end{aligned}
$$




$$
\begin{aligned}
\Lambda(-3) & =4 \zeta \tilde{\zeta} \prod_{i=1}^{N} \rho_{1}\left(-\theta_{i}\right), \\
\left.\bar{\Lambda}(u)\right|_{u \rightarrow \infty} & =\left\{\left(2+c_{1} \tilde{c}_{2}+c_{2} \tilde{c}_{1}\right)^{2}+\left(1+c_{1} c_{2}\right)\left(1+\tilde{c}_{1} \tilde{c}_{2}\right)\right\} u^{2 N+4}+\cdots, \\
\bar{\Lambda}(0) & =\frac{5}{2^{4}}\left(1+c_{1} c_{2}-4 \zeta^{2}\right)\left(1+\tilde{c}_{1} \tilde{c}_{2}-4 \tilde{\zeta}^{2}\right) \prod_{i=1}^{N}\left(\frac{5}{2}-\theta_{i}\right)\left(\frac{5}{2}+\theta_{i}\right), \\
\bar{\Lambda}(-3) & =\frac{5}{2^{4}}\left(1+c_{1} c_{2}-4 \zeta^{2}\right)\left(1+\tilde{c}_{1} \tilde{c}_{2}-4 \tilde{\zeta}^{2}\right) \prod_{i=1}^{N}\left(\frac{5}{2}-\theta_{i}\right)\left(\frac{5}{2}+\theta_{i}\right), \\
\bar{\Lambda}\left(-\frac{1}{2}\right) & =\frac{5}{4} \frac{\zeta \tilde{\zeta}}{\prod_{i=1}^{N}\left(1-\theta_{i}\right)\left(1+\theta_{i}\right)} \Lambda(-1), \\
\bar{\Lambda}\left(-\frac{5}{2}\right) & =\frac{5}{4} \frac{\zeta \tilde{\zeta}}{\prod_{i=1}^{N}\left(1-\theta_{i}\right)\left(1+\theta_{i}\right)} \Lambda(-2) .
\end{aligned}
$$

The $6 N+8$ relations (3.37)-(3.47) enable us completely to determine the eigenvalues $\Lambda(u)$ and $\bar{\Lambda}(u)$ which are given in terms of some inhomogeneous $T-Q$ relations. For simplicity, we first define some functions:

$$
\begin{aligned}
& Z_{1}(u)= \frac{1}{2^{2}} \frac{(u+1)(u+3)}{\left(u+\frac{1}{2}\right)\left(u+\frac{3}{2}\right)} \prod_{j=1}^{N} a\left(u-\theta_{j}\right) a\left(u+\theta_{j}\right) \frac{Q^{(1)}(u-1)}{Q^{(1)}(u)} h_{1}(u) \tilde{h}_{1}(u), \\
& Z_{2}(u)= \frac{1}{2^{2}} \frac{u(u+3)}{\left(u+\frac{1}{2}\right)\left(u+\frac{3}{2}\right)} \prod_{j=1}^{N} b\left(u-\theta_{j}\right) b\left(u+\theta_{j}\right) \frac{Q^{(1)}(u+1) Q^{(2)}\left(u-\frac{3}{2}\right)}{Q^{(1)}(u) Q^{(2)}\left(u+\frac{1}{2}\right)} h_{1}(u) \tilde{h}_{1}(u), \\
& Z_{3}(u)= \frac{1}{2^{2}} \frac{u(u+3)}{\left(u+\frac{3}{2}\right)\left(u+\frac{5}{2}\right)} \prod_{j=1}^{N} b\left(u-\theta_{j}\right) b\left(u+\theta_{j}\right) \\
& \times \frac{Q^{(1)}(u+1) Q^{(2)}\left(u+\frac{5}{2}\right)}{Q^{(1)}(u+2) Q^{(2)}\left(u+\frac{1}{2}\right)} h_{2}(u+3) \tilde{h}_{2}(u+3), \\
& Z_{4}(u)= \frac{1}{2^{2}} \frac{u(u+2)}{\left(u+\frac{3}{2}\right)\left(u+\frac{5}{2}\right)} \prod_{j=1}^{N} e\left(u-\theta_{j}\right) e\left(u+\theta_{j}\right) \frac{Q^{(1)}(u+3)}{Q^{(1)}(u+2)} h_{2}(u+3) \tilde{h}_{2}(u+3), \\
& Q^{(m)}(u)= \prod_{k=1}^{L_{m}}\left(u-\lambda_{k}^{(m)}+\frac{m}{2}\right)\left(u+\lambda_{k}^{(m)}+\frac{m}{2}\right), \quad m=1,2, \\
& f_{1}(u)= \frac{1}{2^{2}} \frac{u(u+1)(u+3)}{\left(u+\frac{3}{2}\right)} \prod_{j=1}^{N} b\left(u-\theta_{j}\right) b\left(u+\theta_{j}\right)\left(u-\theta_{j}+1\right)\left(u+\theta_{j}+1\right) \\
& \times \frac{Q^{(2)}\left(u-\frac{1}{2}\right) Q^{(2)}\left(u-\frac{3}{2}\right)}{Q^{(1)}(u)} h_{1}(u) \tilde{h}_{1}(u) x, \\
& f_{2}(u)= \frac{1}{2^{2}} \frac{u(u+2)(u+3)}{\left(u+\frac{3}{2}\right)} \prod_{j=1}^{N} b\left(u-\theta_{j}\right) b\left(u+\theta_{j}\right)\left(u-\theta_{j}+2\right)\left(u+\theta_{j}+2\right) \\
& Q^{(1)}(u+2)\left(u+\frac{3}{2}\right) \\
& h_{2}(u+3) \tilde{h}_{2}(u+3) x,
\end{aligned}
$$


where $x$ is a constant related to the boundary parameters (see below (3.55)) and $\left\{h_{i}(u), \tilde{h}(u) \mid i=1,2\right\}$ are some functions given by

$$
\begin{array}{lll}
h_{1}(u)=2\left(\sqrt{1+c_{1} c_{2}} u+\zeta\right), & & h_{2}(u)=2\left(\sqrt{1+c_{1} c_{2}} u-\zeta\right), \\
\tilde{h}_{1}(u)=-2\left(\sqrt{1+\tilde{c}_{1} \tilde{c}_{2}} u-\tilde{\zeta}\right), & \tilde{h}_{2}(u)=-2\left(\sqrt{1+\tilde{c}_{1} \tilde{c}_{2}} u+\tilde{\zeta}\right) .
\end{array}
$$

The eigenvalues $\Lambda(u)$ and $\bar{\Lambda}(u)$ can be expressed as

$$
\begin{aligned}
\Lambda(u)= & Z_{1}(u)+Z_{2}(u)+Z_{3}(u)+Z_{4}(u)+f_{1}(u)+f_{2}(u) \\
\bar{\Lambda}(u)= & \prod_{i=1}^{N}\left[\left(u-\theta_{i}+\frac{3}{2}\right)\left(u+\theta_{i}+\frac{3}{2}\right) \tilde{\rho}_{0}\left(u-\theta_{i}+\frac{1}{2}\right) \tilde{\rho}_{0}\left(u+\theta_{i}+\frac{1}{2}\right)\right]^{-1} \\
& \times \frac{1}{2^{4}} \rho_{1}(2 u+3)\left(u-\frac{1}{2}\right)^{-1}\left(u+\frac{3}{2}\right)^{-2}\left(u+\frac{7}{2}\right)^{-1} \\
& \times\left\{Z_{1}\left(u+\frac{1}{2}\right)\left[Z_{2}\left(u-\frac{1}{2}\right)+Z_{3}\left(u-\frac{1}{2}\right)+Z_{4}\left(u-\frac{1}{2}\right)+f_{2}\left(u-\frac{1}{2}\right)\right]\right. \\
& +\left[Z_{2}\left(u+\frac{1}{2}\right)+Z_{3}\left(u+\frac{1}{2}\right)+f_{1}\left(u+\frac{1}{2}\right)\right] Z_{4}\left(u-\frac{1}{2}\right)+Z_{2}\left(u+\frac{1}{2}\right) f_{2}\left(u-\frac{1}{2}\right) \\
& \left.+f_{1}\left(u+\frac{1}{2}\right) Z_{3}\left(u-\frac{1}{2}\right)+f_{1}\left(u+\frac{1}{2}\right) f_{2}\left(u-\frac{1}{2}\right)\right\},
\end{aligned}
$$

where the non-negative integers $L_{1}$ and $L_{2}$ satisfy the constraint ${ }^{1}$

$$
L_{1}=2 L_{2}+N+1
$$

Because the eigenvalues $\Lambda(u)$ and $\bar{\Lambda}(u)$ are both polynomials of $u$, the regularity of these functions gives the constraints of Bethe roots

$$
\begin{aligned}
& \frac{\left(\lambda_{k}^{(1)}+\frac{1}{2}\right)}{\lambda_{k}^{(1)}} \frac{1}{\prod_{j=1}^{N}\left(\lambda_{k}^{(1)}-\theta_{j}-\frac{1}{2}\right)\left(\lambda_{k}^{(1)}+\theta_{j}-\frac{1}{2}\right)} \frac{Q^{(1)}\left(\lambda_{k}^{(1)}-\frac{3}{2}\right)}{Q^{(2)}\left(\lambda_{k}^{(1)}-2\right)} \\
& +\frac{\left(\lambda_{k}^{(1)}-\frac{1}{2}\right)}{\lambda_{k}^{(1)}} \frac{1}{\prod_{j=1}^{N}\left(\lambda_{k}^{(1)}-\theta_{j}+\frac{1}{2}\right)\left(\lambda_{k}^{(1)}+\theta_{j}+\frac{1}{2}\right)} \frac{Q^{(1)}\left(\lambda_{k}^{(1)}+\frac{1}{2}\right)}{Q^{(2)}\left(\lambda_{k}^{(1)}\right)} \\
& +x\left(\lambda_{k}^{(1)}+\frac{1}{2}\right)\left(\lambda_{k}^{(1)}-\frac{1}{2}\right) Q^{(2)}\left(\lambda_{k}^{(1)}-1\right)=0, \quad k=1,2, \cdots, L_{1}, \\
& \frac{1}{\left(\lambda_{l}^{(2)}-1\right)} \frac{Q^{(2)}\left(\lambda_{l}^{(2)}-3\right)}{Q^{(1)}\left(\lambda_{l}^{(2)}-\frac{3}{2}\right)} h_{1}\left(\lambda_{l}^{(2)}-\frac{3}{2}\right) \tilde{h}_{1}\left(\lambda_{l}^{(2)}-\frac{3}{2}\right) \quad \\
& +\frac{1}{\left(\lambda_{l}^{(2)}+1\right)} \frac{Q^{(2)}\left(\lambda_{l}^{(2)}+1\right)}{Q^{(1)}\left(\lambda_{l}^{(2)}+\frac{1}{2}\right)} h_{2}\left(\lambda_{l}^{(2)}+\frac{3}{2}\right) \tilde{h}_{2}\left(\lambda_{l}^{(2)}+\frac{3}{2}\right)=0, \quad l=1,2, \cdots, L_{2} .
\end{aligned}
$$

\footnotetext{
${ }^{1}$ It is remarked that the number of Bethe roots for non-diagonal boundary case is different from (actually more than) that of diagonal boundary case [35]. In the non-diagonal case, there exists a constraint (3.52) between $L_{1}$ and $L_{2}$ because one of the two U(1) symmetry is broken. This leads to the eigenvalue should be given by an inhomogeneous T-Q relation (3.50). But for the diagonal case, the boundaries still preserve the two U(1) symmetries and the eigenvalue can be expressed in terms of a homogeneous T-Q relation [35].
} 
We note that the Bethe ansatz equations obtained from the regularity of $\Lambda(u)$ are the same as those obtained from the regularity of $\bar{\Lambda}(u)$. The function $Q^{(m)}(u)$ has two zero points, namely, $\lambda_{k}^{(m)}-\frac{m}{2}$ and $-\lambda_{k}^{(m)}-\frac{m}{2}$. We have checked that the Bethe ansatz equations obtained from these two points are the same. $x$ is fixed by the asymptotic behaviors of $\Lambda(u)$ and $\bar{\Lambda}(u)$ as

$$
x=\frac{2+c_{1} \tilde{c}_{2}+c_{2} \tilde{c}_{1}}{\sqrt{\left(1+c_{1} c_{2}\right)\left(1+\tilde{c}_{1} \tilde{c}_{2}\right)}}-2 .
$$

If $c_{1}=c_{2}=\tilde{c}_{1}=\tilde{c}_{2}=0$, the boundary reflection matrices reduce to diagonal ones. In this case, $x=0$ and our solution of $\Lambda(u)$ (after taking the homogeneous limit $\left\{\theta_{j} \rightarrow 0 \mid j=\right.$ $1,2, \cdots, N\})$ naturally reduces to that obtained via algebraic Bethe method [35]. Finally, the eigenvalue $E$ of Hamiltonian (3.6) can be expressed in terms of the Bethe roots as

$$
E=\left.\frac{1}{2} \frac{\partial \ln \Lambda(u)}{\partial u}\right|_{u=0,\left\{\theta_{j}\right\}=0} .
$$

\section{Discussion}

In this paper, we generalize the nested ODBA method to the integrable models related to the $s p(4)$ Lie algebra. By using the fusion technique, we obtain the closed operator product identities of the fused transfer matrices. Based on them and the asymptotic behaviors as well as the values of transfer matrices at some special points of $u$, we obtain the exact solution of the system with the periodic and off-diagonal open boundary conditions. The method developed in this paper can be generalized to the high rank $C_{n}$ (i.e., the $s p(2 n)$ ) case directly. ${ }^{2}$

\section{Acknowledgments}

The financial supports from the National Program for Basic Research of MOST (Grant Nos. 2016YFA0300600 and 2016YFA0302104), the National Natural Science Foundation of China (Grant Nos. 11434013, 11425522, 11547045, 11774397, 11775178, 11775177 and 91536115), the Major Basic Research Program of Natural Science of Shaanxi Province (Grant Nos. 2017KCT-12, 2017ZDJC-32), Australian Research Council (Grant No. DP 190101529) and the Strategic Priority Research Program of the Chinese Academy of Sciences, and the Double First-Class University Construction Project of Northwest University are gratefully acknowledged. GL Li would like to thank Shaanxi Province Key Laboratory of Quantum Information and Quantum Optoelectronic Devices, Xian Jiaotong University for its support. The authors also would like to thank F. Wen, P. Sun and Y. Qiao for their valuable discussions.

Open Access. This article is distributed under the terms of the Creative Commons Attribution License (CC-BY 4.0), which permits any use, distribution and reproduction in any medium, provided the original author(s) and source are credited.

\footnotetext{
${ }^{2}$ Although the method in this paper can be generalized to the high rank $C_{n}$ straightforward, it still takes some tedious calculations.
} 


\section{References}

[1] R.J. Baxter, Exactly Solved Models in Statistical Mechanics, Academic Press, New York U.S.A. (1982).

[2] C.-N. Yang, Some exact results for the many body problems in one dimension with repulsive delta function interaction, Phys. Rev. Lett. 19 (1967) 1312 [InSPIRE].

[3] F.C. Alcaraz, M.N. Barber, M.T. Batchelor, R.J. Baxter and G.R.W. Quispel, Surface Exponents of the Quantum XXZ, Ashkin-Teller and Potts Models, J. Phys. A 20 (1987) 6397 [INSPIRE].

[4] E.K. Sklyanin, Boundary Conditions for Integrable Quantum Systems, J. Phys. A 21 (1988) 2375 [INSPIRE].

[5] V.E. Korepin, N.M. Bogoliubov and A.G. Izergin, Quantum Inverse Scattering Method and Correlation Function, Cambridge University Press, Cambridge U.K. (1993).

[6] J. Cao, H.-Q. Lin, K.-J. Shi and Y. Wang, Exact solution of XXZ spin chain with unparallel boundary fields, Nucl. Phys. B 663 (2003) 487 [InSPIRE].

[7] R.I. Nepomechie, Solving the open XXZ spin chain with nondiagonal boundary terms at roots of unity, Nucl. Phys. B 622 (2002) 615 [hep-th/0110116] [InSPIRE].

[8] P. Baseilhac, The q-deformed analogue of the Onsager algebra: Beyond the Bethe ansatz approach, Nucl. Phys. B 754 (2006) 309 [math-ph/0604036] [INSPIRE].

[9] P. Baseilhac and K. Koizumi, Exact spectrum of the XXZ open spin chain from the q-Onsager algebra representation theory, J. Stat. Mech. 2007 (2007) P09006.

[10] H. Frahm, A. Seel and T. Wirth, Separation of Variables in the open XXX chain, Nucl. Phys. B 802 (2008) 351 [arXiv:0803.1776] [INSPIRE].

[11] P. Baseilhac and S. Belliard, Generalized q-Onsager algebras and boundary affine Toda field theories, Lett. Math. Phys. 93 (2010) 213 [arXiv:0906.1215] [INSPIRE].

[12] P. Baseilhac and S. Belliard, The half-infinite XXZ chain in Onsager's approach, Nucl. Phys. B 873 (2013) 550 [arXiv:1211.6304] [INSPIRE].

[13] R.I. Nepomechie, An inhomogeneous T-Q equation for the open $X X X$ chain with general boundary terms: completeness and arbitrary spin, J. Phys. A 46 (2013) 442002 [arXiv:1307.5049] [INSPIRE].

[14] S. Belliard and N. Crampé, Heisenberg XXX Model with General Boundaries: Eigenvectors from Algebraic Bethe Ansatz, SIGMA 9 (2013) 072 [arXiv:1309.6165].

[15] S. Belliard, Modified algebraic Bethe ansatz for XXZ chain on the segment - I: Triangular cases, Nucl. Phys. B 892 (2015) 1 [arXiv:1408.4840] [InSPIRE].

[16] S. Belliard and R.A. Pimenta, Modified algebraic Bethe ansatz for XXZ chain on the segment - II: General cases, Nucl. Phys. B 894 (2015) 527 [arXiv:1412.7511] [InSPIRE].

[17] J. Avan, S. Belliard, N. Grosjean and R.A. Pimenta, Modified algebraic Bethe ansatz for XXZ chain on the segment - III: Proof, Nucl. Phys. B 899 (2015) 229 [InSPIRE].

[18] J. Cao, W. Yang, K. Shi and Y. Wang, Off-diagonal Bethe ansatz and exact solution of a topological spin ring, Phys. Rev. Lett. 111 (2013) 137201 [arXiv:1305.7328] [INSPIRE].

[19] Y. Wang, W.-L. Yang, J. Cao and K. Shi, Off-Diagonal Bethe Ansatz for Exactly Solvable Models, Springer Press, New York U.S.A. (2015). 
[20] X. Zhang, J. Cao, W.-L. Yang, K. Shi and Y. Wang, Exact solution of the one-dimensional super-symmetric $t-J$ model with unparallel boundary fields, J. Stat. Mech. 2004 (2014) P04031.

[21] K. Hao, J. Cao, G.-L. Li, W.-L. Yang, K. Shi and Y. Wang, Exact solution of the Izergin-Korepin model with general non-diagonal boundary terms, JHEP 06 (2014) 128 [arXiv: 1403.7915] [INSPIRE].

[22] J. Cao, W.-L. Yang, K. Shi and Y. Wang, Nested off-diagonal Bethe ansatz and exact solutions of the $\mathrm{SU}(N)$ spin chain with generic integrable boundaries, JHEP 04 (2014) 143 [arXiv: 1312.4770] [INSPIRE].

[23] N.Yu. Reshetikhin, The functional equation method in the theory of exactly soluble quantum systems, Sov. Phys. JETP. 57 (1983) 691.

[24] M.J. Martins and P.B. Ramos, The Algebraic Bethe ansatz for rational braid-monoid lattice models, Nucl. Phys. B 500 (1997) 579 [hep-th/9703023] [INSPIRE].

[25] M. Karowski, On the Bound State Problem in (1+1)-dimensional Field Theories, Nucl. Phys. B 153 (1979) 244 [INSPIRE].

[26] P.P. Kulish, N. Yu. Reshetikhin and E.K. Sklyanin, Yang-Baxter Equation and Representation Theory. 1., Lett. Math. Phys. 5 (1981) 393 [INSPIRE].

[27] P.P. Kulish and E.K. Sklyanin, Quantum spectral transform method recent developments, Lecture Notes Phys. 151 (1982) 61.

[28] A.N. Kirillov and N.Yu. Reshetikhin, Exact solution of the Heisenberg XXZ model of spin s, J. Sov. Math. 35 (1986) 2627.

[29] A.N. Kirillov and N.Y. Reshetikhin, Exact solution of the integrable XXZ Heisenberg model with arbitrary spin. I. The ground state and the excitation spectrum, J. Phys. A 20 (1987) 1565 [INSPIRE].

[30] L. Mezincescu and R.I. Nepomechie, Analytical Bethe Ansatz for quantum algebra invariant spin chains, Nucl. Phys. B 372 (1992) 597 [hep-th/9110050] [INSPIRE].

[31] Y.-k. Zhou, Row transfer matrix functional relations for Baxter's eight vertex and six vertex models with open boundaries via more general reflection matrices, Nucl. Phys. B 458 (1996) 504 [hep-th/9510095] [INSPIRE].

[32] H.J. de Vega and A. González-Ruiz, Exact solution of the $S U-q(n)$ invariant quantum spin chains, Nucl. Phys. B 417 (1994) 553 [hep-th/9309022] [INSPIRE].

[33] H.J. de Vega and A. González-Ruiz, Exact Bethe Ansatz solution for A(n-1) chains with nonSU-q(n) invariant open boundary conditions, Mod. Phys. Lett. A 09 (1994) 2207.

[34] G.-L. Li, R.-H. Yue and B.-Y. Hou, Nested Bethe ansatz for Perk-Schultz model with open boundary conditions, Nucl. Phys. B 586 (2000) 711 [INSPIRE].

[35] G.-L. Li, K.J. Shi and R.H. Yue, Algebraic Bethe Ansatz Solution to $C_{N}$ Vertex Model with Open Boundary Conditions, Commun. Theor. Phys. 44 (2005) 89. 